\title{
Generation of electromotive force in igneous rocks subjected to non-uniform loading*
}

\author{
Akihiro Takeuchi ${ }^{1, *}$ Ömer Aydan ${ }^{2}$ \\ Keizo Sayanagi ${ }^{1}$ and Toshiyasu Nagao ${ }^{1}$ \\ ${ }^{1}$ Earthquake Prediction Research Center, Institute of Oceanic Research and Development, \\ Tokai University, Shizuoka 424-8610, Japan \\ ${ }^{2}$ Department of Marine Civil Engineering, Faculty of Marine Science and Technology, \\ Tokai University, Shizuoka 424-8610, Japan
}

\begin{abstract}
When one end of an air-dry igneous rock block was uniaxially loaded in laboratory, there appeared an electromotive force that made electric currents flow from the stressed volume to the unstressed volume. Quartz-free rocks such as gabbro also generated this force, stronger than quartz-bearing rocks such as granite. This indicates that the piezoelectric effect of quartz and the electrokinetic effect of pore water do not make a large contribution toward generating the electromotive force. We focus on peroxy bond that is one of the abundant lattice defects in igneous rock-forming minerals. When mechanical loading deforms the lattice structure around this defect and breaks its bond, its energy levels change and act like an accepter. As an electron is trapped at this defect from a neighbor $\mathrm{O}^{2-}$ site, a positive hole is activated there. They attempt to diffuse toward the unstressed volume through the valence band and are simultaneously affected by the attractive electric force with the electrons trapped in peroxy bonds. This leads to a polarization in the stressed volume and the generation of electromotive force between the stressed and unstressed volumes. Similar electromotive force may be generated in the Earth's crust where inhomogeneous stress/strain is changing.
\end{abstract}

Key words: electromotive force; igneous rock; non-uniform loading; positive hole CLC number: P319.2 Document code: A

\section{Introduction}

Since early reports by Terada (1931) and Musha (1931) for example, there are a number of reports and reviews that there seems to be abnormal electromagnetic phenomena in the lithosphere, atmosphere, and ionosphere before major earthquakes from data analyses after occurrences of these earthquakes. It has been expected that successful detection of such phenomena before earthquakes is useful for earthquake predictions (e.g., Uyeda et al., 2009). For example, Varotsos and coworkers have tried prediction from anomalous changes of electrotelluric potential difference in Greece (e.g., Varotsos, 2005), and Moriya and coworkers have tried prediction from

\footnotetext{
* Received 27 April 2011; accepted in revised form 10 August 2011; published 10 December 2011.

† Corresponding author. e-mail: atakeuchi@sems-tokaiuniv.jp

(C) The Seismological Society of China and Springer-Verlag Berlin Heidelberg 2011
}

anomalous transmissions of VHF-band radio waves in Hokkaido, north Japan (e.g., Moriya et al., 2010).

Based on a general idea that earthquakes are failure phenomena of focal zones in the Earth's crust to release a portion of its deformation energy, electromagnetic phenomena before earthquakes must be driven by a mechanism preceding failure. On the other hand, the stress/strain in the Earth's crust is not uniform. Especially, it is expected that the distributions in and around focal zones are complicated and must gradually change just before failure (e.g., Kuroki et al., 2003). Considering them, it is expected that the mechanism of electromagnetic phenomena before earthquakes be strongly related to non-uniform changes of the stress/strain conditions. Here, we study electric signals from rock blocks subjected to non-uniform loading in laboratory.

In section 2, we conduct two types of non-uniform loading tests of rock blocks and measure electromotive forces generated in the blocks. In section 3, we conduct 
analyses of the stress/strain distribution in the blocks by means of finite element method (FEM). In section 4 , we discuss mechanisms of the results and application of the results in the laboratory scale to seismoelectromagnetic phenomena in the geophysical scale.

\section{Non-uniform loading tests}

\subsection{Electric currents induced by non-uniform loading on various rocks}

The rocks used in the first series tests are (1) gabbro from South Africa, (2) medium-grained granite from Japan, (3) fine-grained granite from Japan, and (4) white marble from Taiwan of China. There seemed no preferred orientation of the grain-shape/distribution in the rocks. The rocks were cut into block-shape with a size of $2.5 \mathrm{~cm} \times 3.0 \mathrm{~cm} \times 10 \mathrm{~cm}$. The surfaces were polished with abrasives \#800. Thereafter, the blocks were dried in air for more than one week.

Figure 1 shows a schematic of the experimental set-up. The blocks were placed inside an aluminum enclosure acting as a Faraday cage. One end of the blocks was uniaxially loaded by means of a manual hydraulic press (RIKEN, CDM-20PA). The load was measured with a load cell (Minebea, CMM1-2T and CSD-819C). The strain along the loading axis was measured by a strain gauge (Minebea, F-3N-12T11W03) on the loaded end surface. Copper tapes with graphite-based conductive adhesive were pasted on the loaded areas $(2.5 \times 2.5$ $\mathrm{cm}^{2}$ each) and the unloaded end side $\left(2.5 \times 3.0 \mathrm{~cm}^{2}\right)$ as electrodes. The loading areas and the aluminum rectangular pistons for loading were grounded. The unloaded end was grounded through an electrometer (ADCMT, 8240 ) with the impedance of about $30 \mathrm{M} \Omega$ in case of a picoampere-range ammeter.

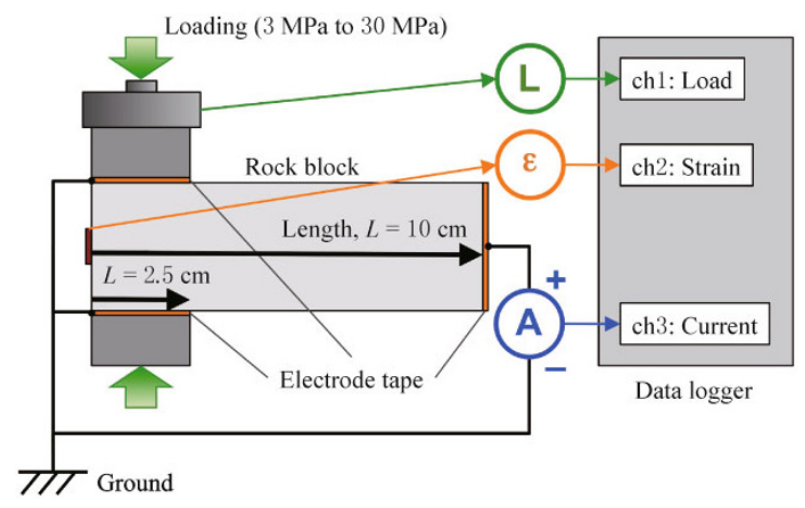

Figure 1 A schematic of the experimental set-up for non-uniform loading of an air-dry rock block (height 3.0 $\mathrm{cm}$, length $10 \mathrm{~cm}$, thickness $2.5 \mathrm{~cm}$ ).
The blocks were first loaded at $3 \mathrm{MPa}$ for more than $10 \mathrm{~min}$. This was due to the lower limit of the load cell and to eliminate the effect of the block slightly moving at the application of the first load from 0 MPa. Then, a linear loading up to $30 \mathrm{MPa}$ was superimposed with a load rate of $(1 \pm 0.15) \mathrm{MPa} / \mathrm{s}$. Data of the load, strain, and current were analog out-put to a recorder (HIOKI, 8808-50) and recorded at $16 \mathrm{~Hz}$ sampling. Loading tests were repeated many times with samples of all types of rocks.

Figure 2 shows an example of the experimental results in case of fine-grained granite. The positive value in the stress means compression. The positive value in the strain means contraction. The current value indicated is the difference from the value at $3 \mathrm{MPa}$. The positive value in the current means flowing from the unloaded end to the ground through the electrometer (the clockwise flow of current in Figure 1). The current
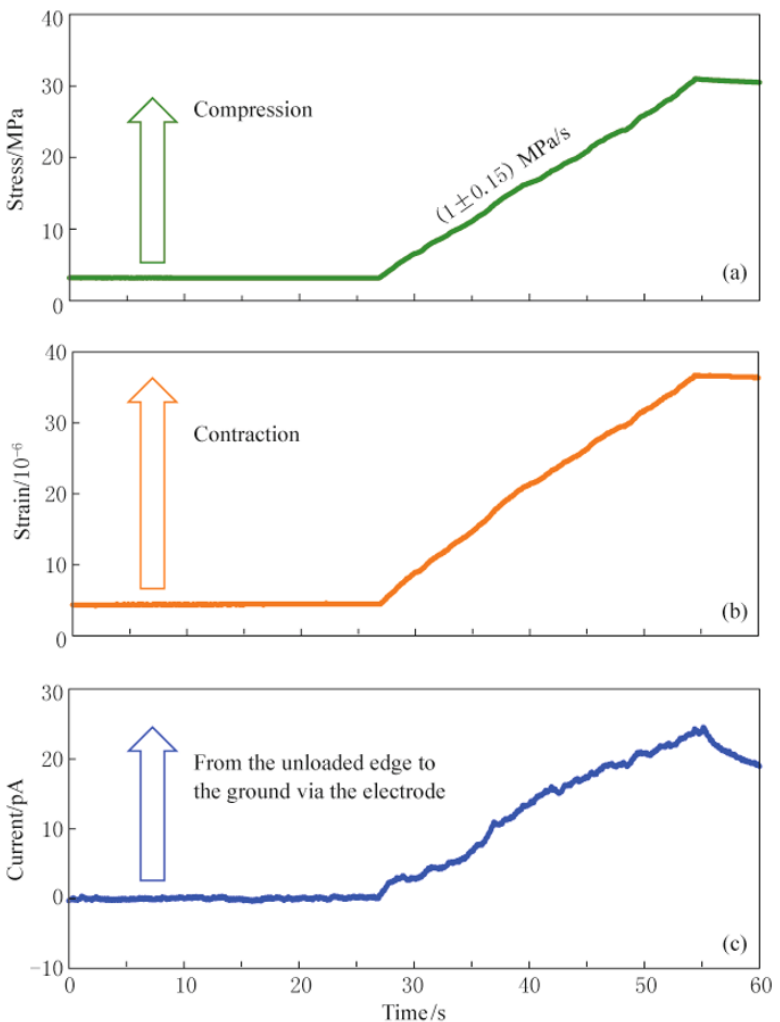

Figure 2 An example of the experimental results for fine-grained granite. (a) Stress: from $3 \mathrm{MPa}$ to $30 \mathrm{MPa}$ at $(1 \pm 0.15) \mathrm{MPa} / \mathrm{s}$, positive value corresponding to compression. (b) Strain: positive value corresponding to contraction. (c) Current: difference from the value at 3 $\mathrm{MPa}$, positive value corresponding to the current from the unloaded edge to the ground through the electrode (the clockwise flow of current in Figure 1). 
amplitude linearly increased with increase of load and strain, at least, in the range we subjected in this experiments. Gabbro and medium-grained granite also generated similar currents, but white marble did not generate any remarkable current. Figure 3 shows the current intensity at $30 \mathrm{MPa}$ for all kinds of rocks. Quartz-free gabbro generates currents stronger than quartz-rich granite and much stronger than non-igneous marble. Actually, the amplitude of marble's current was nearly equal to the background noise level and should be considered to be zero.

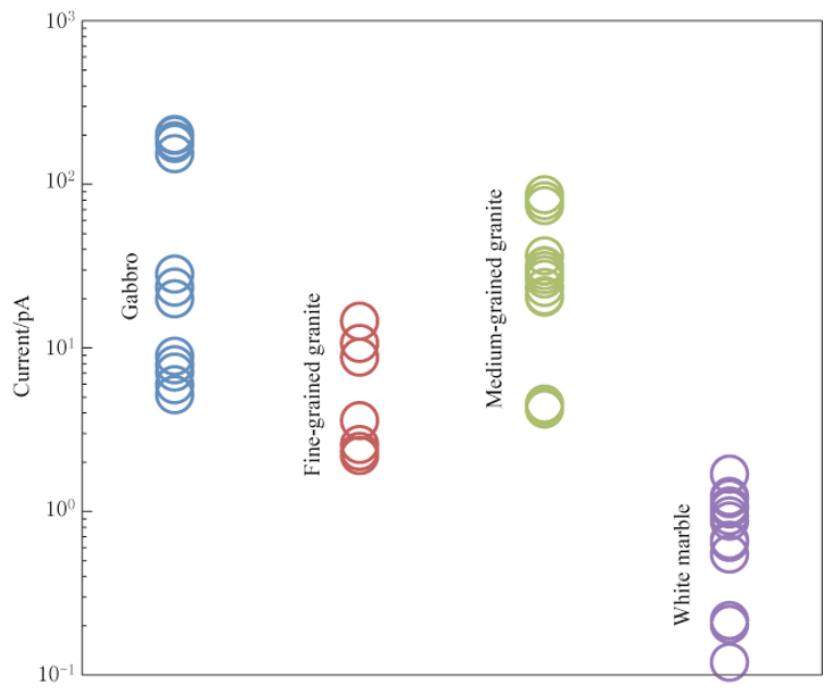

Figure 3 The current amplitude at the stress of 30 $\mathrm{MPa}$ for all types of rocks. The currents mean difference from the value at $3 \mathrm{MPa}$.

\subsection{Electromotive force versus block length}

The rocks used in the second series tests are gabbro and fine-grained granite, used also in the first tests. The rocks were cut into block-shape with a size of 2.5 $\mathrm{cm} \times 3.0 \mathrm{~cm} \times L \mathrm{~cm}(2.5 \leq L \leq 10)$. The surfaces were polished and the blocks were air-dried in the same ways. The experimental set-up of the second tests was same as that of the first tests (Figure 1). In case of the shortest block $(L=2.5 \mathrm{~cm})$, the whole volume of the block was uniaxially loaded. The same load profile was used in all tests. In the second tests, the potential difference was also measured with the electrometer. Its impedance was more than $10^{13} \Omega$ in case of a voltmeter mode. Loading tests were repeated with many samples of two types of rocks. Here, note that the current and the potential difference were measured separately.

Figure 4 shows an example of the experimental results in case of gabbro. The positive value in the stress means compression. The positive value in the strain
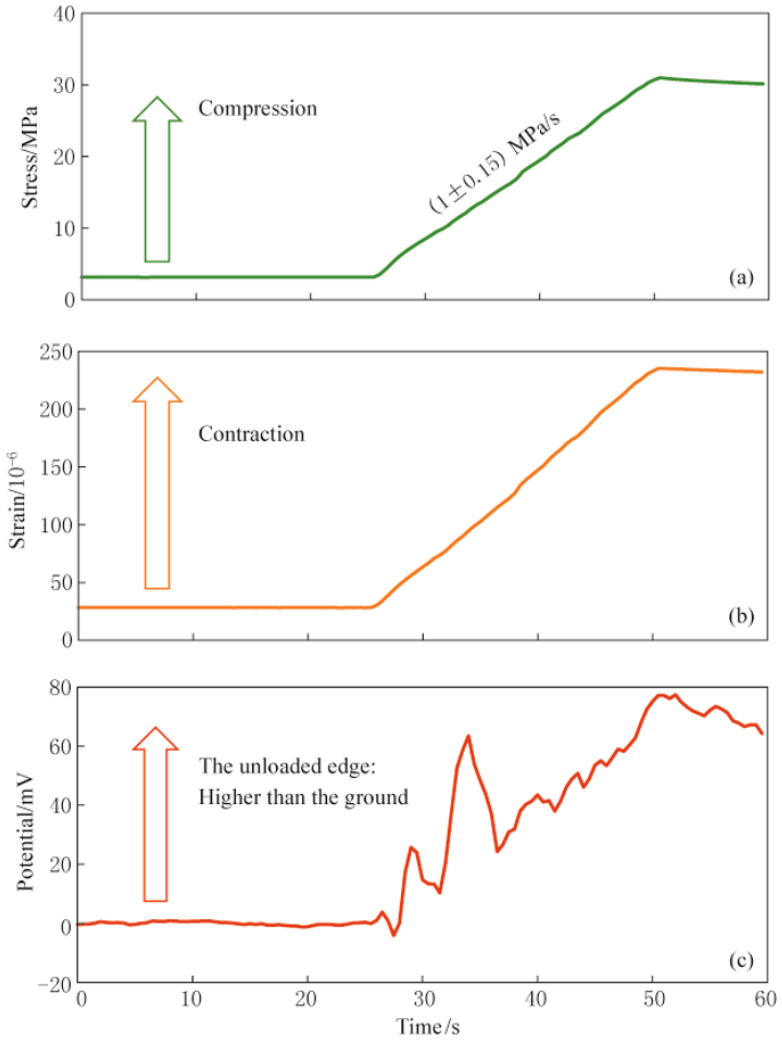

Figure 4 An example of the experimental results for gabbro. (a) Stress: from $3 \mathrm{MPa}$ to $30 \mathrm{MPa}$ at $1 \pm 0.15 \mathrm{M}-$ $\mathrm{Pa} / \mathrm{s}$, positive value corresponding to compression. (b) Strain: positive value corresponding to contraction. (c) Potential difference: difference from the value at $3 \mathrm{MPa}$, positive value corresponding the electric potential high at the unloaded end relative to the ground, making the clockwise flow of current in Figure 1.

means contraction. The potential difference value indicated is the difference from the value at $3 \mathrm{MPa}$. The positive value in the potential difference means an electromotive force making a current flow from the unloaded end to the ground through the electrometer (clockwise in Figure 1). This was concordant with the results in case of the current measurements. Similar to the current, the amplitude of potential difference linearly increased with increases of the load and strain. In many cases, the potential difference (and the current also) fluctuates a little with loading increasing. However, a large fluctuation shown in Figure 4c is rare although this is probably not a measurement error. Fine-grained granite also generated similar currents and potential differences. Figure 5 shows the amplitude of the currents and potential differences at $30 \mathrm{MPa}$ for the two types of rocks. Quartz-free gabbro also generates currents and potential differences stronger than quartz-rich granite 
(a) Gabbro

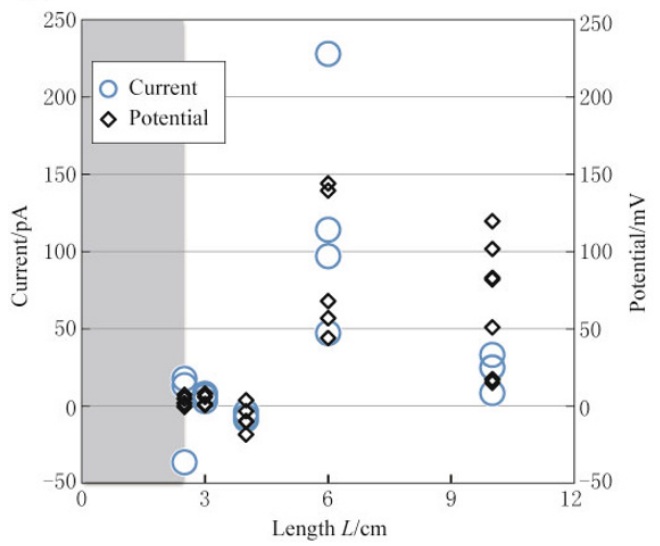

(b) Fine-grained granite

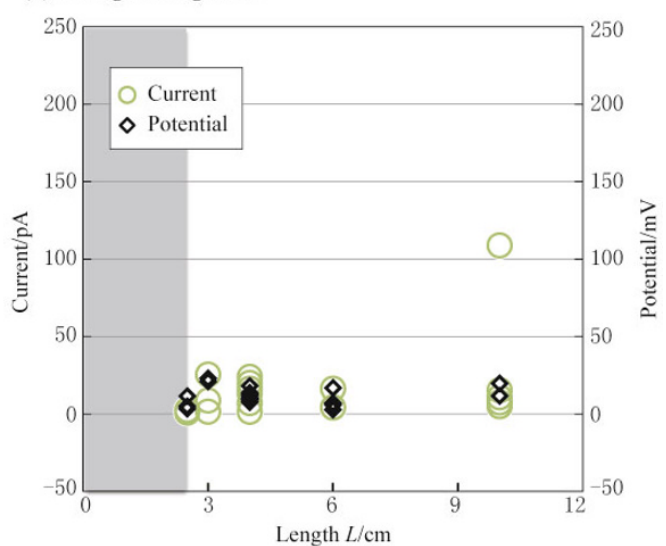

Figure 5 All results of the current and potential difference at the stress of $30 \mathrm{MPa}$ of rock blocks with various lengths for gabbro (a) and fine-grained granite (b). The currents and potentials are difference from the value at $3 \mathrm{MPa}$.

in all of the length ranges. These amplitudes are roughly zero at the length $L=2.5 \mathrm{~cm}$ and become the maximum in the range of $4 \mathrm{~cm} \leq L \leq 6 \mathrm{~cm}$ although this trend is not so clear for granite.

\section{Finite element method (FEM) analyses}

To understand dynamics of rock samples subjected to non-uniform loading, we also conducted analyses of

(a)

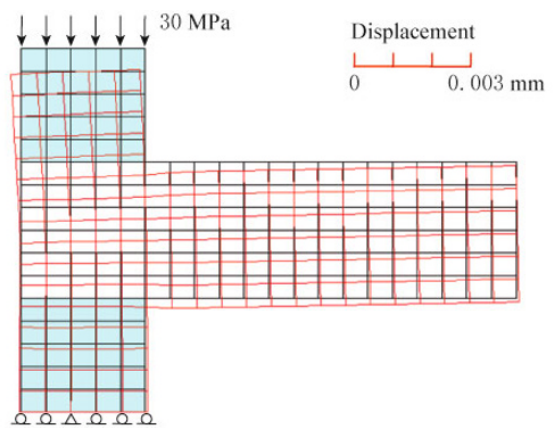

(b)

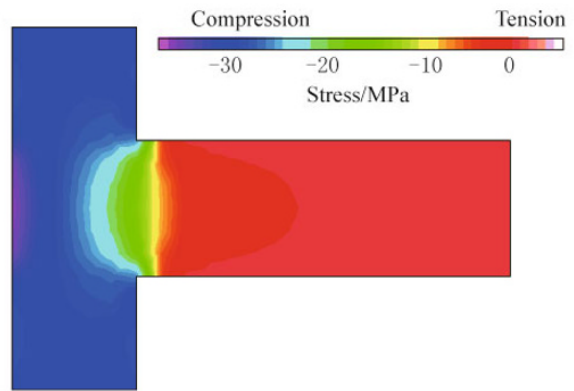

the stress/strain distributions in the samples by means of finite element method (FEM). Figure 6a shows the finite element mesh and boundary conditions (in black) for $2 \mathrm{D}$ analyses of the stress/strain distributions in a rock block subjected to non-uniform loading. The sample simulated was a $10 \mathrm{~cm}$ long gabbro block $(L=10$ $\mathrm{cm})$ with a height of $3 \mathrm{~cm}$ and a thickness of $2.5 \mathrm{~cm}$. This sample was loaded through a pair of the aluminum rectangular pistons at one of its ends. The sample and rectangular pistons were assumed to be in full contact

(c)
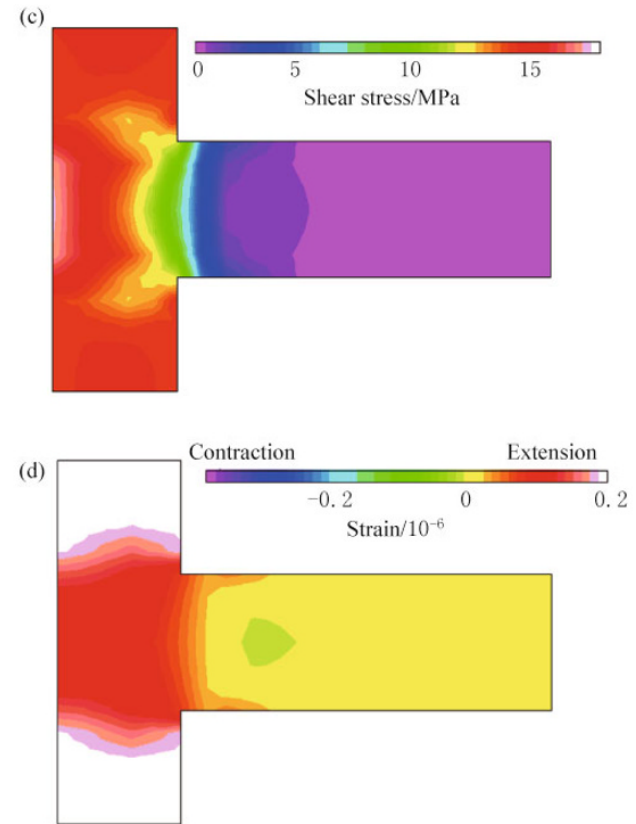

Figure 6 Finite element analyses of a gabbro block with the length of $10 \mathrm{~cm}$ under the stress of 30 MPa. (a) The finite element mesh and boundary conditions (in black) and the deformed configuration (in red). (b) The distribution of the third principal stress. (c) The distribution of the maximum shear stress. (d) The distribution of the horizontal strain component. 
without any relative slip. The center of the bottom surface of the lower rectangular pistons was fixed horizontally and vertically. A uniformly distributed $30 \mathrm{MPa}$ traction was applied to the top surface of the upper rectangular pistons. The gabbro block and rectangular pistons were discretized using four-noded iso-parametric finite elements with a side length of $5 \mathrm{~mm}$. They were assumed to behave linear elastically under the assumed loading condition. Elastic moduli of gabbro and rectangular pistons were set as $100 \mathrm{GPa}$ and $70 \mathrm{GPa}$, respectively. Poisson's ratio was 0.25 for the gabbro sample and 0.35 for the rectangular pistons. FEM computations were carried out using a multi-purpose elastoplastic finite element code developed for rock engineering structures incorporating interface elements for discontinuities and special elements for support systems also (Aydan et al., 1988, 2011; Kawamoto and Aydan, 1999).

Figure 6a shows the deformed configuration (in red) overlapping with the original mesh (in black). Figures $6 \mathrm{~b}, 6 \mathrm{c}$, and $6 \mathrm{~d}$ show the distributions of the third principal stress, the maximum shear stress, and the horizontal strain component, respectively. The positive values imply tension while the negative values compression. These computational results clearly indicated that the sample was highly deformed at the loaded end while the load-free volume $(5 \mathrm{~cm}<L \leq 10 \mathrm{~cm})$ was almost stress/strain free.

\section{Discussion}

\subsection{Possible candidates for the cause of elec- tromotive force}

Piezoelectric effect of quartz (e.g., Finkelstein et al., 1973; Nitsan, 1977; Yoshida et al., 1997; Yoshida and Ogawa, 2004) is the first possible candidate for the electromotive force because quartz is involved in many types of rocks and piezovoltage could be so strong under certain conditions. However, this effect cannot explain the electromotive force detected in gabbro that is quartz free. Of course, this effect should contribute to the force detected in two types of granite in this study. However, its contribution will not be the prime cause because an apparent piezoelectric coefficient of granite is generally three orders of the magnitude smaller than that of single crystal quartz (Sasaoka et al., 1998).

Electrokinetic effect of pore water (e.g., Mizutani et al., 1976; Fitterman, 1978; Jouniaux and Pozzi, 1997) is the second possible candidate. In general, pore water includes a number of ions. In case of the mineral-water system, positive ions are generally predominant in the water, while negative ions adhere on the mineral surfaces, although depending on $\mathrm{pH}$ of water (Ishido and Mizutani, 1981). Flow of such water is equivalent to flow of electric current. This flow from the loaded volume to the unloaded volume could make the electric potential of the unloaded volume high. However, this effect could not explain the electromotive force detected in the rock samples that were well air-dried.

Rock-forming minerals involve a lot of lattice defects. They are the third possible candidates. For example, Vallianatos and coworkers uniaxially loaded the whole volume of white marble blocks and measured electric currents with a pair of electrode plates conductively pasted on sample sides perpendicular to the load axis (e.g., Stavrakas et al. 2003; Vallianatos et al., 2004). Although the amplitude of currents was very small under low stress levels, it increased when the stress level reached about $60 \%-80 \%$ of its failure stress. They explained these currents based on the motion of charged dislocations (e.g., Slifkin, 1993) and named 'pressure stimulated currents'. Aydin and coworkers also conducted similar tests and obtained the results concordant with those of Vallianatos's group (Aydin et al., 2009). Their results seem to be concordant with our results of white marble samples subjected to non-uniform $30 \mathrm{MPa}$ loading (roughly about $30 \%$ of the failure stress). If we loaded the marble samples stronger, we might detect obvious electric currents. However, to explain the electromotive forces detected in stiff igneous rock samples in our tests, a number of dislocations - much larger content than that in marble - should be positively charged and move from the stressed volume toward the unstressed volume much faster than those in marble under low stress levels. This is not concordant with the nature of dislocations; dislocations cannot move fast under low temperatures and low pressures.

Finally, we focus on peroxy bond $\left(\mathrm{O}_{3} \mathrm{X}-\mathrm{OO}-\mathrm{YO}_{3}\right.$, with $\mathrm{X}, \mathrm{Y}=\mathrm{Si}^{4+}, \mathrm{Al}^{3+}$, etc.), a type of the lattice defects in rock-forming minerals. When mechanical loading deforms the lattice structure around this defect and breaks its bond, an unoccupied energy level of the bond shifts down from forbidden band toward valence band and an electron can jump in this level from a neighboring oxygen site. This results in an activation of a positive hole at the neighbor and a trap of an electron at the broken bond. Once activated, positive holes can spread away through the valence band, like charge carriers in a ptype semiconductor (e.g., Freund, 2010; Takeuchi et al., 2010). 
The concentration of activated positive holes will increase with an increase of the stress/strain level, at least, in the range we subjected. Considering the distributions of stress/strain as shown in Figure 6, there will distribute activated positive holes and trapped electrons with a gradation that is the highest at the loaded end $(L=0 \mathrm{~cm})$ and zero at the middle of the block $(4 \mathrm{~cm}<L<6 \mathrm{~cm})$. Because positive holes are mobile, they attempt to diffuse toward the unstressed volume. At the same time, they are electrically attracted by the trapped electrons (Figure 7). As a result, an electric unevenness, i.e. an electric polarization, is formed in the stressed volume $(L<4 \mathrm{~cm})$. This causes the electromotive force between the loaded and unloaded ends. In case of medium long samples $(4 \mathrm{~cm} \leq L \leq 6 \mathrm{~cm})$, electrodes are located at the both terminals of the polarization. On the other hand, in case of longer samples $(L \geq 6 \mathrm{~cm})$, the positive electrode is far from the polarization. This is why the electromotive force was the strongest in case of medium long samples (Figure 3). When the ammeter mode electrode connected the unloaded edge and the ground, electrons flowed in the wire from the ground to the unloaded edge to cancel the polarization. As a result, the ammeter detected a current flowing from the edge to the ground through the ammeter (the clockwise flow of current in Figure 1).

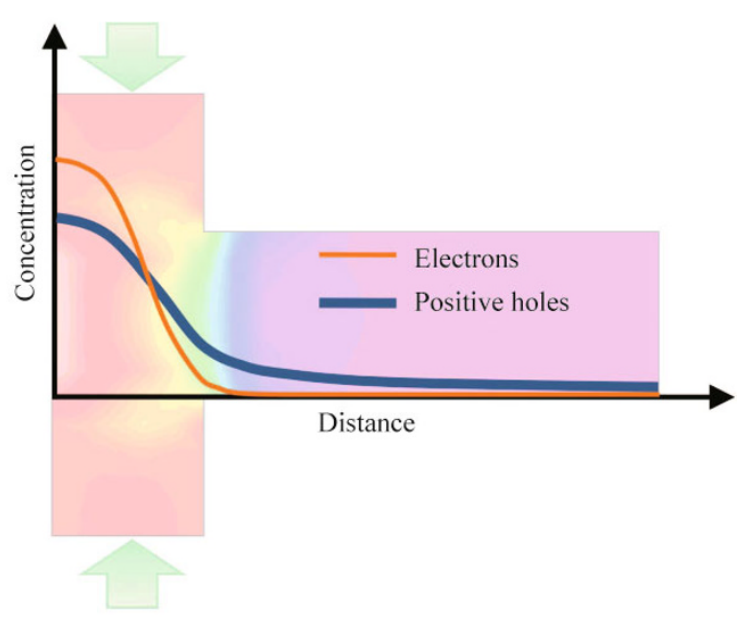

Figure 7 Schematic distributions of the electrons and positive holes overlapping the distribution of the maximum shear stress in a rock block subjected to nonuniform loading.

Because the source of igneous rocks, i.e. magma, generally involves water, igneous rocks involve traces of water as hydroxyl impurities during high-temperature crystallization. Some of the impurities are subsequent- ly converted to peroxy bonds plus $\mathrm{H}_{2}$ molecules during cooling. This process is universal on Earth. Therefore, peroxy bond is generally involved in all types of minerals forming igneous rocks and high-grade metamorphic rocks. On the other hand, because white marble is mostly formed by calcite in which the conversion from hydroxyl pairs to peroxy bonds plus $\mathrm{H}_{2}$ molecules does not occur, the stress-activation of positive holes is not driven in white marble. Thus, peroxy bond seems to be the most reasonable candidate to explain the electromotive force induced in igneous rocks subjected to non-uniform loading.

\subsection{Application to seismo-electromagnetic phe- nomena}

In this section, we try a simple application of the experimental results to seismo-electromagnetic phenomena in the geophysical scale. When a local change in the crustal stress appears in a focal zone before faulting, it is expected that holes be activated in and around the focal zones (Figure 8). This situation would be similar to the non-uniform loading test of the blocks with the length $6 \mathrm{~cm} \leq L \leq 10 \mathrm{~cm}$. If we could install one terminal of the electrodes in the hypocenter, we would effectively measure abnormal changes in the electric potential difference between the hypocenter and the Earth surface.

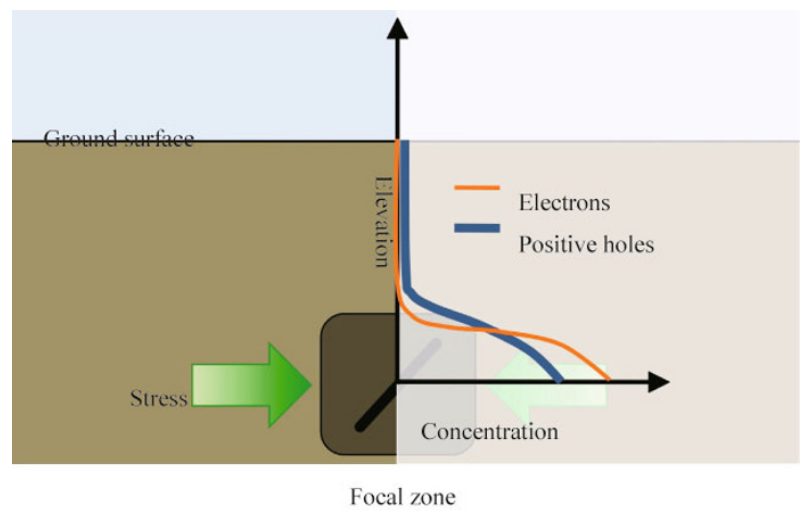

Figure 8 Schematic distributions of the electrons and positive holes overlapping with the right half of a schematic of the Earth's crust in and around a focal zone.

If the Earth's crust is dry, a portion of the positive holes activated in the focal zone will reach the ground surface. They will easily form a vertical electric field on the order of $\mathrm{kV} / \mathrm{m}$ only over distances on the order of tens of nanometers from the ground surface. This field can ionize the air (most likely $\mathrm{O}_{2} \rightarrow \mathrm{O}_{2}^{+}$) and inject massive amounts of positive air ions into the lower atmosphere. Diffusion of these ions upward changes the 
ordinal atmospheric electric field and causes electric attraction of ions in the ionosphere downward (Freund et al., 2009). When a vertical atmospheric electric field formed by the positive holes on the ground surface is enough strong, say $\sim 1 \mathrm{kV} / \mathrm{m}$ macroscopically on the ground level in a wide area, it may directly perturb plasma in the ionosphere (Takeuchi et al., 2006). On the other hand, if the Earth's crust is wet due to pore water, positive holes will be caught in the pore water. They will combine with negative ions or oxidize water molecules (i.e., $\mathrm{H}_{2} \mathrm{O}_{2}$ ) and lead further chemical reactions (Balk et al., 2009; Grant et al., 2011). Thus, a portion of the dynamic energy in focal zones is converted to the electric energy, and a portion of the electric energy is further converted to the chemical energy. Thus, there should be complicated dynamic-electromagneticchemical linkages in the Earth's crust. These linkages cause various types of phenomena before earthquakes.

\section{Summary}

Gabbro, medium-grained granite, fine-grained granite, and white marble were cut into the rectangular block (height $3.0 \mathrm{~cm}$, length $10 \mathrm{~cm}$, thickness $2.5 \mathrm{~cm}$ ). After air-dry for more than one week, one end of each block was uniaxially loaded up to $30 \mathrm{MPa}$. The contact range for loading was up to $2.5 \mathrm{~cm}$ away from the loaded end (the contact area was $2.5 \times 2.5 \mathrm{~cm}^{2}$ ). An electrometer, which connected the unloaded end and the ground, detected electric currents from the unloaded end to the ground during loading. Gabbro and two types of granites generated the currents much stronger than marble. Moreover, gabbro tended to generate the currents and potential differences stronger than two types of granites.

Gabbro and fine-grained granite were cut into the shape of a rectangular block with various lengths (height $3.0 \mathrm{~cm}$, length $2.5 \mathrm{~cm} \leq L \leq 10 \mathrm{~cm}$, thickness 2.5 $\mathrm{cm})$. After air-dry for more than one week, one end of each block was uniaxially loaded up to $30 \mathrm{MPa}$. The contact range for loading was up to $2.5 \mathrm{~cm}$ away from the loaded end (the contact area was $2.5 \times 2.5 \mathrm{~cm}^{2}$ ). An electrometer, which connected the unloaded end and the ground, detected electric currents from the unloaded end to the ground during loading and electric potentials that were high at the unloaded end relative to the ground. Their amplitudes become the maximum at the length $4 \mathrm{~cm} \leq L \leq 6 \mathrm{~cm}$. Gabbro generates the currents and potential differences stronger than finegrained granite in all length ranges.

According to FEM analyses of the stress/strain distribution in a gabbro block under the same non- uniform loading condition, the stress/strain intensity was the maximum at the loaded end and decreased to zero at the length $4 \mathrm{~cm}<L<6 \mathrm{~cm}$. The gradation of the stress/strain distribution ranging $0 \leq L<6 \mathrm{~cm}$ seems to relate to the maximum intensity of the current/potential at the length $4 \mathrm{~cm}<L<6 \mathrm{~cm}$.

We focus on peroxy bonds $\left(\mathrm{O}_{3} \mathrm{X}-\mathrm{OO}-\mathrm{YO}_{3}\right.$, with $\mathrm{X}, \mathrm{Y}=\mathrm{Si}^{4+}, \mathrm{Al}^{3+}$, etc.), an abundant lattice defect in igneous rock-forming minerals. When mechanical loading deforms the lattice structure around this defect and breaks its bond, an unoccupied energy level of the bond shifts down from forbidden band toward valence band and an electron can jump in this level from a neighboring oxygen site. This results in an activation of a positive hole at the neighbor and a trap of an electron at the broken bond. The concentration of activated positive holes will increase with the rate at which deviatoric stresses are applied, at least, in the range we subjected in this experiment. So that, there will be distributions of activated positive holes and trapped electrons with a gradation that is the highest at the loaded end $(L=0$ $\mathrm{cm}$ ) and zero at the middle of the block $(4 \mathrm{~cm}<L<6$ $\mathrm{cm})$. Because positive holes are mobile, they attempt to diffuse toward the unstressed volume. They are simultaneously attracted from the trapped electrons. As a result, an electric polarization is formed in the stressed volume. This was detected as the electromotive force that made currents flow from the unstressed edge to the ground via the electrometer.

When a local change in the crustal stress appears in a focal zone before failure, it is expected that holes be activated in and around the focal zones. If we could install an electrode in the focal zone, we would effectively measure abnormal changes in the electric potential difference between the focal zone and the ground surface. However, there are pore water and underground water in the actual Earth's crust. Although water may shut out transmission of positive charges, chemical reactions between them will cause new chemical phenomena. We can say that not only failure but also such energy conversions are ways of energy release for focal zones.

Acknowledgements This study is partially supported by "Observation and Research Program for Prediction of Earthquakes and Volcanic Eruptions" of the Ministry of Education, Culture, Sports, Science and Technology of Japan, "FY2010 Research Incentive Assistance Program" of Educational System General Research Organization, Tokai University, and "Individual Research Projects" of Institute of Oceanic Development 
of Science and Technology, Tokai University. Finally, we thank two reviewers for their helpful comments and suggestions on our manuscript.

\section{References}

Aydan Ö, Kyoya T, Ichikawa Y, Kawamoto T, Ito T and Shimizu Y (1988). Three-dimensional simulation of an advancing tunnel supported with forepoles, shotcrete, steel ribs and rockbolts. In: Swoboda G eds. Numerical Methods in Geomechanics, Innsbruck 1988. Proceedings of the Sixth International Conference on Numerical Methods in Geomechanics. Balkema Publications, Rotterdam, Netherlands, 1 481-1 486.

Aydan Ö, Uehara F and Kawamoto T (2011). A numerical study on the long-term performance of an underground powerhouse subjected to varying initial stress state, cyclic water head and temperature variations. International Journal of Geomechanics (in press).

Aydin A, Prance R J, Prance H and Harland C J (2009). Observation of pressure stimulated voltages in rock using an electric potential sensor. Appl Phys Lett 95(12): 124102 .

Balk M, Bose M, Ertem G, Rogoff D A, Rothschild L J and Freund F T (2009). Oxidation of water to hydrogen peroxide at the rock-water interface due to stress-activated electric currents in rocks. Earth Planet Sci Lett 283(1-4): 87-92.

Finkelstein D, Hill R D and Powell J R (1973). The piezoelectric theory of earthquake lightning. $J$ Geophys Res 78(6): 992-993.

Fitterman D V (1978). Electrokinetic and magnetic anomalies associated with dilatant regions in a layered earth. $J$ Geophys Res 83(B12): 5 923-5 928.

Freund F T (2010). Toward a unified solid state theory for pre-earthquake signals. Acta Geophysica 58(5): 719-766.

Freund F T, Kulahci I G, Cyr G, Ling J, Winnick M, Tregloan-Reed J and Freund M M (2009). Air ionization at rock surfaces and pre-earthquake signals. Journal of Atmospheric and Solar-Terrestrial Physics 71(17-18): $1824-1834$.

Grant R A, Halliday T, Balderer W P, Leuenberger F, Newcomer M, Cyr G and Freund F T (2011). Ground water chemistry changes before major earthquakes and possible effects on animals. International Journal of Environmental Research and Public Health 8(6): 1 936-1 956.

Ishido T and Mizutani H (1981). Experimental and theoretical basis of electrokinetic phenomena in rock-water systems and its applications to geophysics. Journal of Geophysics 86(B3): 1 763-1 775.

Jouniaux L and Pozzi J-P (1997). Laboratory measurements anomalous $0.1-0.5 \mathrm{~Hz}$ streaming potential under geochemical changes: Implications for electrotelluric precursors to earthquakes. J Geophys Res 102(B7): 15 33515343.

Kawamoto T and Aydan Ö (1999). A review of numerical analysis of tunnels in discontinuous rock masses. International Journal for Numerical and Analytical Methods in Geomechanics 23(13): 1 377-1 391.

Kuroki H, Ito H M and Yoshida A (2003). Strain and stress changes in the Tokai region of central Japan expected from a 3D subduction model. Phys Earth Planet Inter 135(4): 231-252.

Mizutani H, Ishido T, Yokokura T and Ohnishi S (1976). Electrokinetic phenomena associated with earthquakes. Geophys Res Lett 3(7): 365-368.

Moriya T, Mogi T and Takada M (2010). Anomalous preseismic transmission of VHF-band radio waves resulting from large earthquakes, and its statistical relationship to magnitude of impending earthquakes. Geophys J Inter 180(2): 858-870.

Musha K (1931). On the luminous phenomenon that attended the Izu earthquake, November 26th, 1930. Bulletin of Earthquake Research Institute, The University of Tokyo 9: $214-215$.

Nitsan U (1977). Electromagnetic emission accompanying fracture of quartz-bearing rocks. Geophys Res Lett 4(8): 333-336.

Sasaoka H, Yamanaka C and Ikeya M (1998). Measurements of electric potential variation by piezoelectricity of granite. Geophys Res Lett 25(12): 2 225-2 228.

Slifkin L (1993). Seismic electric signals from displacement of charged dislocations. Tectonophysics 224(1-3): 149-152.

Stavrakas I, Anastasiadis C, Triantis D and Vallianatos F (2003). Piezo stimulated currents in marble samples: Precursory and concurrent-with-failure signals. Natural Hazards and Earth System Sciences 3(3-4): 243-247.

Takeuchi A, Lau B W S and Freund F T (2006). Current and surface potential induced by stress-activated positive holes in igneous rocks. Physics and Chemistry of the Earth, Parts A/B/C 31(4-9): 240-247.

Takeuchi A, Futada Y, Okubo K and Takeuchi N (2010). Positive electrification on the floor of an underground mine gallery at the arrival of seismic waves and similar electrification on the surface of partially stressed rocks in laboratory. Terra Nova 22(3): 203-207.

Terada T (1931). On luminous phenomena accompanying earthquakes. Bulletin of Earthquake Research Institute, The University of Tokyo 9: 225-255.

Uyeda S, Nagao T and Kamogawa M (2009). Shortterm earthquake prediction: Current status of seismoelectromagnetics. Tectonophysics 470(3-4): 205-213.

Vallianatos F, Triantis D, Tzanis A, Anastasiadis C and Stavrakas I (2004). Electric earthquake precursors: from laboratory results to field observations. Physics and Chemistry of the Earth, Parts A/B/C 29(4-9): 339-351.

Varotsos P (2005). The Physics of Seismo Electric Signals. Terra Scientific Publishing Company, Tokyo, 338pp.

Yoshida S and Ogawa T (2004). Electromagnetic emissions from dry and wet granite associated with acoustic emissions. J Geophys Res 109(9): B09204.

Yoshida S, Uyeshima M and Nakatani M (1997). Electric potential changes associated with slip failure of granite: Preseismic and coseismic signals. J Geophys Res $\mathbf{1 0 2 ( B 7 ) : ~}$ 14 883-14 897. 